\title{
Brachytherapy of tongue carcinoma in a patient with difficult airway: anesthetic considerations
}

\author{
Zainab Abdul Ghaffar, MBBS, MMedl.2, Soon Eu Chong, MD, MMed ${ }^{2,3}$, Kok Leng Tan, MD, MMed³, \\ Gokula Kumar Appalanaido, MD, FRANZCR 4 , Muhamad Yusri Musa, MD, MMed², Hasmah binti Hussin, MD, MMed 2,3, \\ Nurulhuda Jusoh, MB, BCh, BAO2 \\ Integrative Medicine Cluster, Advanced Medical and Dental Institute, Universiti Sains Malaysia, Penang, ${ }^{2}$ Section of Surgery and Anesthesia. \\ Advanced Medical and Dental Institute, Universiti Sains Malaysia, Penang, ${ }^{3}$ Regenerative Medicine Cluster, Advanced Medical and Dental \\ Institute, Universiti Sains Malaysia, Penang, ${ }^{4}$ Oncology Cluster, Advanced Medical and Dental Institute, Universiti Sains Malaysia, Penang, \\ Malaysia
}

\begin{abstract} patients in future.

\section{Purpose}

With the advancement of technology, brachytherapy for head and neck tumor has becoming more popular and gained a successful role in treatment $[1,2,3]$. It can either be carried out in combination with external beam radiation therapy, surgery, chemotherapy, or as a separate treatment for patients who are unsuitable for tongue resection [4]. To achieve a static position for implantation of the applicators, adequate pain relief and immobilization is compulsory; thus, most of the time, general anesthesia is required [5]. However, there are a few precautions in this group of patients as these patients are prone to certain complications related to their challenging airway, sharing of airway [6], and disease chronicity. We report a case of brachytherapy applicator insertion on a difficult airway patient, where the unsecured airway had resulted in a number of difficult situations after the procedure.
\end{abstract}

The practice of brachytherapy in unresectable tongue carcinoma is gaining popularity. However, this procedure poses specific anesthetic challenges, particularly challenges of airway sharing and a higher rate of difficult airway. We report a 74-year-old chronic smoker, chronic alcoholic with history of stroke, who had undergone brachytherapy for tongue carcinoma. Apart from a huge tongue tumor, he had an epiglottic mass but refused elective tracheostomy. This had led to a few critical states throughout the process of treatment, including a metabolic crisis due to thiamine deficiency and difficult airway crisis. To our best knowledge, there have been no reported case on a patient with vocal cord mass undergoing tongue brachytherapy. We hope sharing of this experience may aid the management of similar

J Contemp Brachytherapy 2018; 10, 6: 573-576 DOI: https://doi.org/10.5114/jcb.2018.79856

Key words: anesthesia, brachytherapy, difficult airway, tongue carcinoma.

\section{Case report}

A 74-year-old men presented with painful and swollen tongue for three months. He was on hypertension and diabetes treatment. He was a chronic alcoholic and a 50-pack-year smoker. Besides, he had history of stroke one year prior, with no residual neurological deficit. The patient was diagnosed with tongue carcinoma but refused curative surgery. He could not tolerate oral food intake and was currently on nasogastric tube feeding. He opted for tongue brachytherapy and the applicator was planned to be inserted under general anesthesia.

The patient's tongue was massive due to its cancerous status (Figure 1). It was extremely tender when touched. Mouth opening was good, there was no stridor on lying flat. However, when indirect laryngoscopy was performed, a fungating lesion was noted on the epiglottis, obscuring the vocal cord (Figure 2). Elective tracheostomy was suggested but was declined by the patient, even though the benefits and risks were explained.

In view of possible difficult intubation, the patient was advised to be well-fasted and proceeded with awake fiberoptic intubation. The nose was anaesthetized with cocaine $4 \%$ prior to the procedure. Under conscious sedation with dexmedetomidine infusion, he was successfully intubated. Tongue, epiglottic mass biopsy, and brachytherapy implant was inserted successfully (Figure 3). Since the patient had refused elective tracheostomy, he was extubated fully awake after the procedure, and was 

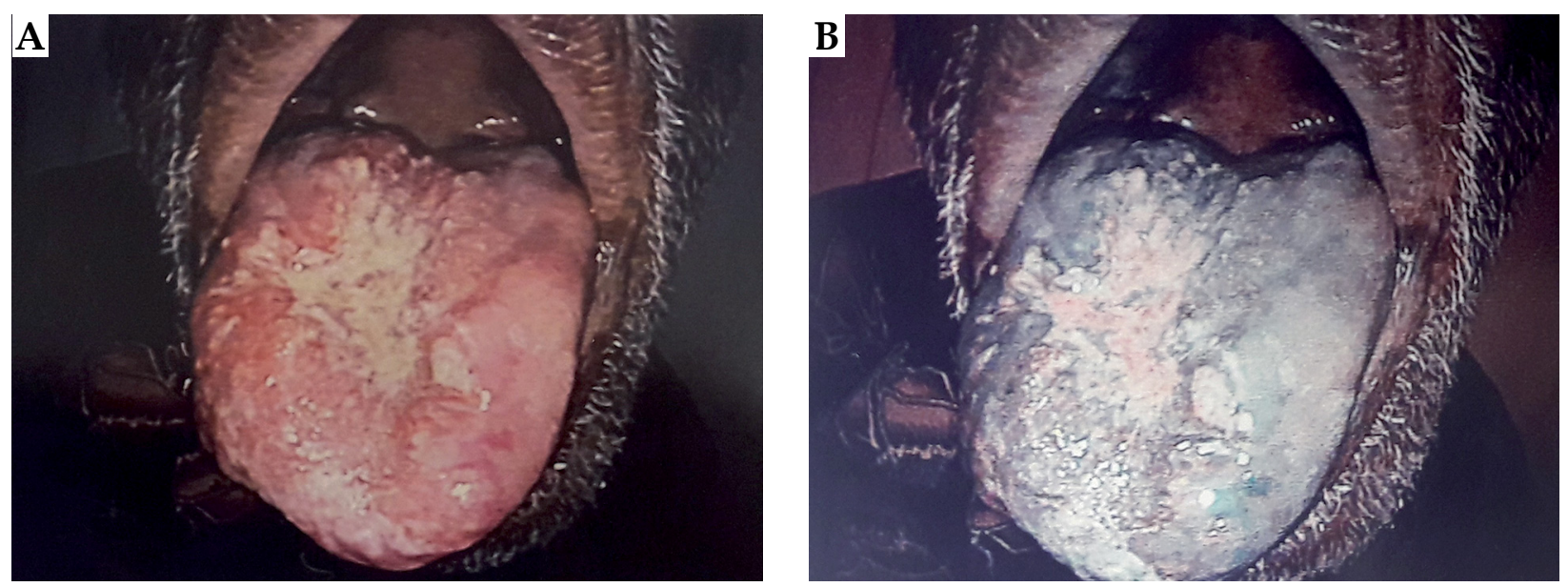

Fig. 1. The tongue was huge and extremely tender when touched. A) Mallampati score was III. B) A better visualization of neoplastic changes under narrow-band imaging system

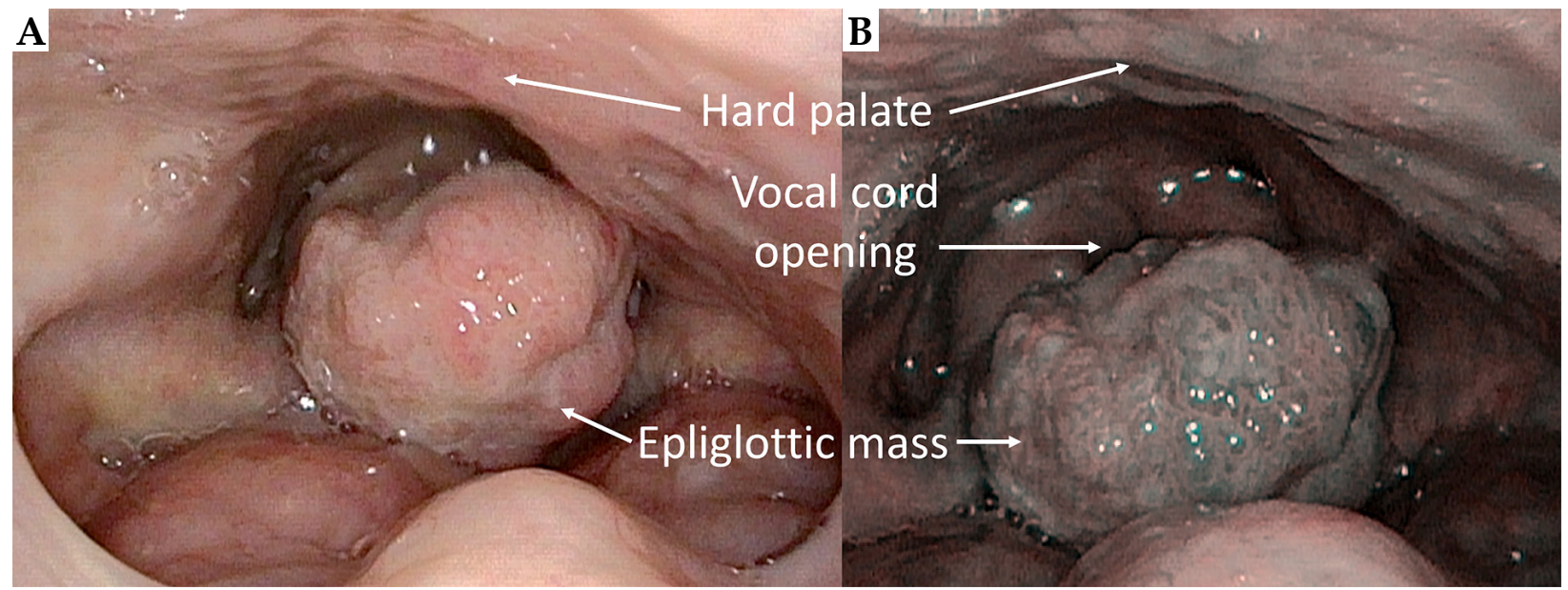

Fig. 2. Fungating lesion arising from the epiglottis, altering the normal epiglottis anatomy. A) Mass obscuring the vocal cord.

B) Image under narrow-band imaging endoscopy system

admitted to high dependency unit for close observation in view of his multiple comorbidities.

Seven hours after extubation, the patient developed delirium with tremors, restlessness, ophthalmoplegia, and nystagmus. His blood pressure (BP), heart rate (HR), and oxygen saturation $\left(\mathrm{SpO}_{2}\right)$ were stable. Blood investigations were all normal, except a high lactate level (7.8 $\mathrm{mmol} / \mathrm{l})$. He attempted to remove the brachytherapy applicators and intravenous cannula but was stopped by the nurses. Intramuscular haloperidol $5 \mathrm{mg}$ was given two times as well as intravenous (IV) midazolam $1 \mathrm{mg}$; however, the patient returned to delirious state short after the sedative effect of drugs had wean off. Difficult airway alert was activated. Due to the big tongue tumor and the unremoved epiglottic mass, further administration of anxiolytic and sedative medications may cause over-sedation leading to a catastrophic upper airway obstruction. After a multidisciplinary discussion, a diagnosis of encephalopathy due to thiamine deficiency was established. IV thiamine and alprazolam tablet $0.25 \mathrm{mg}$ were administered. Soon after the medications, his condition improved. He became calm and coopera- tive. Repeat lactate levels reduced and normalized within 6 hours. He was discharged to general ward after two days. Brachytherapy was carried out for two weeks followed by removal of the brachytherapy applicators.

However, during the removal of tongue brachytherapy implant under local anesthesia, bleeding occurred from the applicator insertion site, which required compression. This caused severe pain to the patient. He refused to cooperate, and bleeding was hard to control. It was even harder to remove the deep-seated applicators. Upon struggling, one of the applicators had fell off and dropped into the throat of patient. Emergency endoscopy revealed that the applicator was noticed below the vocal cord of the patient. It was then gently removed.

The procedure was temporarily withheld, and the patient was sent to operating theatre for emergency removal of the leftover applicators and cessation of bleeding under general anesthesia. The patient had then agreed for tracheostomy. He was no longer cooperative for awake fiberoptic intubation, thus was induced under inhalational anesthesia. Fortunately, laryngeal mask airway was able to be inserted, while maintaining spontaneous ventila- 
tion. Tracheostomy was fashioned and all brachytherapy applicators were successfully removed.

\section{Discussion}

The authors reported in detail their experience on a patient with tongue carcinoma, difficult airway, and multiple comorbidities, who had undergone brachytherapy. There were no similar cases for reference in literature. Brachytherapy on a high-volume tongue cancer was planned on this patient with an epiglottic mass. There was sharing of airway. The refusal of elective tracheostomy to secure the airway properly had cause the patient and clinicians to experience a few challenging situations.

After brachytherapy applicator insertion, this patient developed encephalopathy due to thiamine deficiency by the evidence of delirium, ophthalmoplegia, and hyperlactatemia, which responded rapidly with administration of IV thiamine. Patients with chronic alcohol consumption, acute metabolic stress, and malignancy have higher risk of developing thiamine deficiency. Thiamine is not synthesized in the human body and requires continuous dietary intake [7]. Chronic alcohol consumption interferes thiamine absorption from the digestive system. Besides, as thiamine plays a fundamental role in energy metabolism, an increased metabolic demand such as fasting, stressful event, and malignancy could deplete thiamine storage causing further deficiency. Lack of thiamine increases lactate production by altering the aerobic metabolism. As tongue carcinoma are usually associated with chronic alcoholism, tobacco usage [8], and malnutrition, such complication should be anticipated. Retrospectively, nutrition status should be optimized perioperatively. Fasting time should be reduced to minimum and feeding with nourishing fluid should be restarted as soon as possible after procedure. The diagnosis of thiamine deficiency should be considered in similar patient with non-hypoxic lactic acidosis.

This patient had suffered a crisis following the delirious state because it was difficult to titrate the sedative medications due to the epiglottic mass just above the vocal cord. An excess of sedation may lead to upper airway obstruction and this can cause 'cannot ventilate cannot intubate' (CVCI) situation. Secondly, the removal of applicator was done under local anesthesia. Difficulty was encountered when removing the deeply seated applicators, as there were contact bleeding and severe pain. All these had caused the patient to become restless and uncooperative. Worst still, it had caused one of the removed applicators to fall into the airway. When the patient was subsequently planned for applicator removal under general anesthesia, he was not cooperative for awake fiberoptic intubation. Fortunately, supraglottic airway was successfully inserted and tracheostomy was done. After the airway was secured with tracheostomy, the removal procedure was carried out easily. The learning point of this crisis was that prophylactic tracheostomy should have been administered prior to the insertion of tongue brachytherapy applicator, according to the American Society of Brachytherapy guidelines $[9,10]$. This applies especially to patients with huge tongue

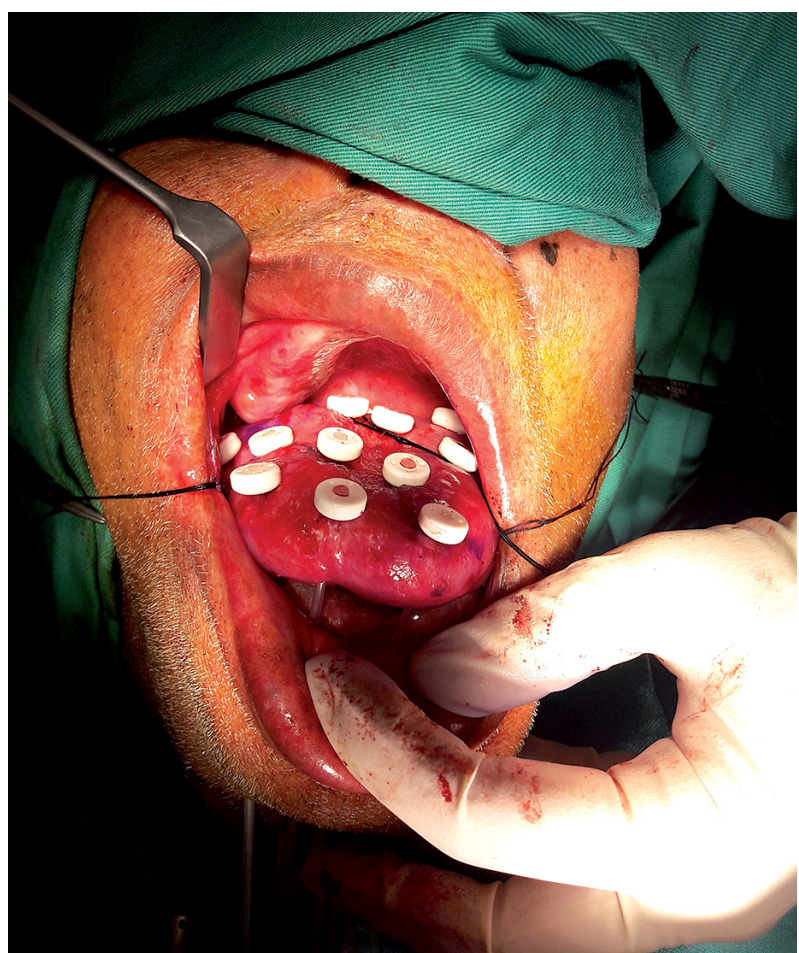

Fig. 3. Brachytherapy applicators were inserted successfully under general anesthesia

tumor or with a potential difficult airway. Thus, any of the crisis following brachytherapy could be managed easily when the airway is well-protected.

\section{Conclusions}

For brachytherapy of head and neck, it is important to have a well-planned strategy involving multidisciplinary input from pre-procedure optimization until recovery. To the best of our knowledge, this is the first reported case on a patient with vocal cord mass undergoing tongue brachytherapy. We intend to share our experience for the management of similar patients in future.

\section{Acknowledgement}

The publication fee of this article was supported by Universiti Sains Malaysia Short Term Grant (304/ CIPPT/6315192). We sincerely thank Lim Jo Anne for critical reading and useful comments of this manuscript.

\section{Disclosure}

Authors report no conflict of interest.

\section{References}

1. Hegde JV, Demanes DJ, Veruttipong D et al. Head and neck cancer reirradiation with interstitial high-dose-rate brachytherapy. Head Neck 2018; 40: 1524-1533.

2. Skowronek J. Current status of brachytherapy in cancer treatment - short overview. J Contemp Brachytherapy 2017; 9: 581-589.

3. Khalilur R, Hayashi K, Shibuya H. Brachytherapy for tongue cancer in the very elderly is an alternative to external beam radiation. Br J Radiol 2011; 84: 747-749. 
4. Chadha J, Hu KS, Jacobson A et al. The role of brachytherapy in treatment of oral tongue cancer. Int J Radiat Oncol Biol Phys 2016; 94: 890.

5. Ambasta S, Parida S, Rudingwa P, Mishra SK. Brachytherapy implant insertion in head-and-neck cancer: Results of anaesthetic technique at a tertiary care hospital. Indian J Anaesth 2018; 62: 470-472.

6. Juneja R, Lacey O. Anaesthesia for head and neck cancer surgery. Current Anaesthesia \& Critical Care 2009; 20: 28-32.

7. Onishi H, Ishida M, Tanahashi I et al. Subclinical thiamine deficiency in patients with abdominal cancer. Palliat Support Care 2018; 16: 497-499.

8. Andrade JO, Santos CA, Oliveira MC. Associated factors with oral cancer: a study of case control in a population of the Brazil's Northeast. Rev Bras Epidemiol 2015; 18: 894-905.

9. Nag S, Cano ER, Demanes DJ et al. The American Brachytherapy Society recommendations for high-dose-rate brachytherapy for head-and-neck carcinoma. Int J Radiat Oncol Biol Phys 2001; 50: 1190-1198.

10. Kovács G, Martinez-Monge R, Budrukkar A et al. GEC-ESTRO ACROP recommendations for head \& neck brachytherapy in squamous cell carcinomas: 1st update - Improvement by cross sectional imaging based treatment planning and stepping source technology. Radiother Oncol 2017; 122: 248-254. 